\title{
Estudio sobre metodologías de enseñanza y competencias en Educación Primaria
}

\author{
Rita Ros Pérez-Chuecos. Universidad de Murcia \\ Recepción: 26 de octubre de 2015 | Revisión: 3 de noviembre de 2015 | Aceptación/Publicación: 11 de noviembre de 2015 \\ Correspondencia: rita.ros@um.es \\ Citar: Ros Perez-Chuecos, R. (2015). Estudio sobre metodologías de enseñanza y competencias en Educación Primaria. ReiDoCrea, \\ 4, 378-385. [http://hdl.handle.net/10481/38788]
}

\begin{abstract}
Resumen: En este trabajo se analizan las metodologías de enseñanzas o técnicas didácticas de un centro de Educación Primaria de la Región de Murcia, con la finalidad de identificar el enfoque predominante (enseñanza tradicional centrado en el docente o todo lo contrario un enfoque por competencias en donde el alumno tiene un papel más activo). El diseño de la investigación es un estudio de casos, con carácter descriptivo, en el que participaron catorce docentes. Los resultados obtenidos sugieren que las clases aún siguen siendo de forma magistral y que las metodologías aplicadas aún están más centradas en el papel del docente que en la gestión activa del alumno. Con la incorporación de las competencias estamos en el camino de una renovación metodológica en donde el trabajo por proyectos en los centros tiene especial relevancia.
\end{abstract}

Palabra clave: Enseñanza | Desarrollo de habilidades

Study on Teaching Methodologies and Skills in Primary Education

Abstract: In this work, teaching methodologies and teaching techniques of a Primary Education of the Region of Murcia, in order to identify the predominant approach (traditional teacher-centered teaching or the opposite approach by competencies analyzes where student has a more active) paper. The research design is a case study, with descriptive, in the fourteen participating teachers. The results suggest that the classes are still being masterfully and methodologies applied are still more focused on the role of teachers in the active management of the student. With the addition of the skills we are in the way of a methodological renewal where project work in schools has special relevance.

Keywords: Teaching | Skills Development

\section{Introducción}

La Educación escolar se ha visto expuesta a grandes transformaciones que inducen a la innovación educativa, así como a la implantación de nuevos enfoques en la praxis pedagógica. Hasta ahora las innovaciones se han centrado en los docentes como agentes de cambio dejando de lado al sujeto de la educación: el alumno.

Con la LOE se establece un nuevo modelo- educativo caracterizado por el papel activo del alumno, en el que las competencias básicas entendidas como "aquellas que debe haber desarrollado un joven o una joven al finalizar la enseñanza obligatoria para poder lograr su realización personal, ejercer la ciudadanía activa, incorporarse a la vida adulta de manera satisfactoria y ser capaz de desarrollar un aprendizaje permanente a lo largo de la vida" (RD 1513/2006, p. 1), cobran especial relevancia. Concretamente se debe de contribuir a la adquisición y desarrollo de las ocho competencias básicas desde un planteamiento integrador e interdisciplinar que permita al alumno alcanzar una realización personal e incorporarse a la vida adulta de manera satisfactoria, desarrollando aprendizajes a lo largo de la vida adulta. Este modelo se mantiene con la LOMCE, pero se matiza el carácter procesual, no concluso, de la competencia.

La investigación sobre enfoques de enseñanza por competencias se ha estudiado principalmente en el contexto universitario a consecuencia del Proceso de Convergencia Europea, pero es extensible a otros niveles. Las competencias constituyen la base fundamental para orientar el currículo, la docencia, el aprendizaje y la evaluación desde un marco de calidad, ya que brinda principios, indicadores y 
herramientas para hacerlo, más que cualquier otro enfoque educativo (Tobon, 2006). Propone un cambio en el proceso de enseñanza aprendizaje en donde el alumno alcance para su desarrollo social, integral y personal un conjunto de destrezas, habilidades, actitudes y valores necesarios para su desarrollo personal y social.

Dado que el objeto de estudio es identificar la metodología docente, resulta esencial determinar qué se entiende por metodología. Herrán (2008) define la metodología como:

"la intención educativa del docente y sus premisas didácticas: concepción de educación, de enseñanza, de su didáctica específica, la idea que tiene del alumno, sus conocimientos aplicados a los elementos curriculares básicos (intenciones docentes, competencias, contenidos, criterios de evaluación), sus valores educativos, su capacidad para gestionar la motivación didáctica de los alumnos, su cota de respeto didáctico, entre otras" (p.4).

En definitiva, la metodología se entiende como la actividad que responde a los modos de proceder desde la práctica. La metodología y la actividad didáctica no se puede comprender una sin la otra porque una actividad implica una técnica didáctica de referencia. Desde las metodologías participativas, interés se centra en la calidad y no la cantidad de los aprendizajes. De ahí que se fomenta el papel activo de los alumnos en su propio aprendizaje, lo que implica autoconocerse y autorregularse, orientando las actividades a tareas reales en contextos flexibles y diversificados.

\section{Método}

\section{Participantes}

En total han participado en la investigación catorce de los treinta y cinco docentes que pertenecen a un Colegio Público rural de la Región de Murcia. Esta muestra corresponde con el $40 \%$ de los docentes que representan el centro educativo. Éstos corresponden con las especialidades de: Educación Infantil (2), Educación Primaria (8), Educación Física (1), Inglés (1), Educación Musical (1) y Religión (1).Se ha tenido en cuenta para su elección que cada uno fuera de nivel educativo y especialidad diferente. De los 14 entrevistados, 9 son mujeres $(64,2 \%)$ y 5 hombres $(35,7)$. El rango de edad de los participantes osciló entre los 25 y los 39 años $(M=34,13)$, con una antigüedad media de 4 años y medio de trabajo en este centro educativo. En relación al nivel educativo el 100,0\% tenían estudios de Diplomatura o Grado, entre los cuales sólo el $16,6 \%$ poseían estudios de Licenciatura, Doctorado o Máster Universitarios, especificando que eran complementos a su formación inicial.

\section{Instrumento y procedimiento}

Para la recogida de la información se ha utilizado una entrevista semiestructurada con preguntas abiertas y cerradas. Esta consta de dos partes; la primera datos identificativos respecto agénero, edad, la titulación (especialidad), el curso al que imparte clase, materias que imparte, años de docente, interinidad o titularidad..., y la segunda parte incluye diferentes preguntas específicas sobre el tema principal de la investigación: competencias, metodologías aplicadas, evaluación, contenidos, utilización del libro de texto, recursos utilizados, coordinación con el resto de docentes... El último apartado está compuesto por un espacio de reflexión para el docente con cuestiones tales como ¿Que le ha parecido la entrevista? ¿Le ha sido de utilidad para su práctica profesional? Instrumento que ha sido validado por un consenso de expertos de diferentes áreas de conocimiento universitarias. 
Los datos se recogieron durante el mes de mayo del año 2015, en horario de mañanas, principalmente en las tutorías de los docentes. La investigación fue presentada por el Director en el Claustro de Profesores de la tarde lo que incitó a que los docentes fueren más receptivos en las entrevistas.

Esta investigación es un estudio de casos con carácter descriptivo cuyo principal objetivo es el análisis de la realidad educativa de un centro. Se trata de una investigación cualitativa que aplica el método de las Ciencias Sociales.

Las metodologías tienen un carácter cuantitativo y han sido tratadas con el paquete estadístico SPSS, versión 15.0, con el cual hemos procedido al cálculo de los estadísticos descriptivos. Con relación a las variables más cualitativas hemos realizado el análisis de los datos textuales recogidos a través de la entrevista y las observaciones. Así se organizó la información de dichos datos para extraer los significados que se encierran en torno al problema de investigación planteado, utilizando la valoración interjueces. En concreto fueron un grupo de 8 expertos de los cuales 5 eran mujeres y 3 hombres, docentes de la Universidad de Murcia, en concreto del departamento de Didáctica y Organización Escolar (DOE). Se utilizaron como criterios para su validación: claridad, pertinencia y adecuación. En el anexo 1 aportamos la entrevista semiestructurada tal y como ha sido realizada a los docentes.

\section{Resultados}

A continuación se procede analizar los datos obtenidos en las entrevistas:

En la primera parte (datos identificativos), llama la atención que el $100 \%$ de los docentes se encuentran en situación de interinidad en el centro, es decir, no son titulares de la plaza. Los docentes tienen edades comprendidas entre 25 y 39 años $(M=34,13)$. De los 14 entrevistados, 9 son mujeres $(64,2 \%)$ y 5 hombres $(35,7)$. Respecto a la especialidad que imparten son diversas: Educación Infantil (2), Educación Primaria (8), Educación Física (1), Inglés (1), Educación Musical (1) y Religión (1). Lo mismo sucede con el curso que imparten, se intentó que la representación fuese lo más heterogénea posible, de ahí que los maestros entrevistados impartan clase en distintos niveles. Escogimos una maestra en Educación Musical, un maestro de Inglés, otro de Educación Física y una maestra de Religión, especialistas que dan clase desde $1^{\circ}$ hasta $6^{\circ}$. Respecto a los docentes de Primaria, 4 son tutores de los niveles, $1^{\circ}, 3^{\circ}, 4^{\circ}, 6^{\circ}$ y 2 de los niveles de $2^{\circ}$ y otros 2 docentes de $5^{\circ}$, aportando de este modo una gran riqueza a la muestra.

En la segunda parte hace referencia a cuestiones diversas:

- Competencias: La gran mayoría de los docentes dicen que las competencias son conseguidas a través de los objetivos, pero no saben especificar una competencia concreta, lo confunden con temas transversales. Explican que se está hablando del mismo término aunque en una época diferente y con un nombre diferente, llegando a la conclusión de que a lo largo de su docencia siempre ha existido este tema y que lo único que hacen es cambiarle el nombre. Sólo dos de los docentes detallan las 8 competencias, llegando a especificar que los datos recogidos aportan información relevante sobre el desarrollo integral del discente (adquisición de destrezas, habilidades, actitudes, valores...), en palabras de los docentes.

- Metodologías: existen diferentes metodologías utilizadas por los docentes en el aula, primando la lección magistral $(57 \%)$ frente al trabajo grupal $(29 \%)$ e individual $(14 \%)$ del alumno. Se concreta que los entrevistados responden a que se utilizan diferentes metodologías de enseñanza dependiendo del trabajo a realizar en el 
aula (figura 1). En el modelo de enseñanza grupal se recogen diversas técnicas didácticas. El $65 \%$ de los entrevistados utilizan el debate $(22 \%)$, la resolución de ejercicios (12\%) y el trabajo dirigido (14\%) como principal tarea a realizar dentro de esta modalidad de enseñanza. En menor medida los docentes trabajan el aprendizaje basado en problemas $(7 \%)$, caso dramatizado $(8 \%)$, juegos educativos $(5 \%)$, dinámica de grupo $(2 \%)$ y juegos de roles $(2 \%)$. Un $11 \%$ especifica en categoría de otros técnicas como: Teaching, pecera, Phillips 66, seminario clásico o prácticas ordenadores. Todo ello supone un total de 60 respuestas relacionadas con el trabajo grupal. Respecto a la técnica de trabajo individual o autónomo connotaciones similares. Todos los entrevistados recogen la enseñanza personalizada como metodología de trabajo en el aula. Casi lo mismo sucede con la enseñanza programada, 13 de los 14 docentes dicen aplicarla en el aula. En menor medida sucede con el aprendizaje basado en problemas (10\%), el aprendizaje orientado a proyectos $(4 \%)$, la enseñanza modular $(2 \%) u$ otros como categoría miscelánea donde un solo docente realiza algún trabajo individual como audio-tutoría (tabla 1). Como se aprecia la metodología aplicada por los docentes permite que el alumno adquiera un papel más activo en su proceso de enseñanzaaprendizaje. Por ello, es necesario utilizar una actividad didáctica que responda al objetivo que se pretende.

Figura 1

Modelos de enseñanza aplicados en el aula

\section{Modelo de enseñanza}

- Lección magistral

- Trabajo en grupo

- Trabajo autónomo

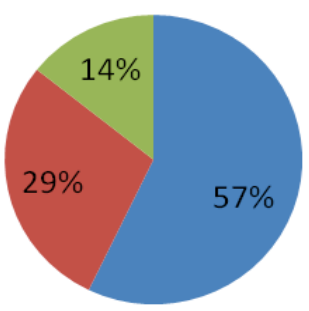

Tabla 1

Metodología de trabajo grupal y autónomo

\begin{tabular}{|lcclccc|}
\hline \multicolumn{1}{c}{ Trabajo en grupo } & $\mathbf{F}$ & $\%$ & \multicolumn{1}{c}{ Trabajo autónomo } & $\mathbf{F}$ & $\%$ \\
\hline Aprendizaje basado en problemas (ABP) & 4 & 7 & Aprendizaje de lecturas & 5 & 10 \\
Caso dramatizado & 5 & 8 & Aprendizaje orientado a proyectos & 2 & 4 \\
Debate & 13 & 22 & Enseñanza modular & 1 & 2 \\
Juegos educativos & 3 & 5 & Enseñanza personalizada & 14 & 28 \\
Resolución de ejercicios & 12 & 20 & Enseñanza programada & 13 & 26 \\
Trabajo dirigido & 14 & 23 & Contrato de aprendizaje & 4 & 8 \\
Dianámica de grupo & 1 & 2 & Programa de lecturas & 7 & 14 \\
Juego de roles & 1 & 2 & Enseñanza a distancia & 3 & 6 \\
Otros & 7 & 11 & Otros: audio-tutoría & 1 & 2 \\
\hline Total & 60 & 100 & Total & 50 & 100 \\
\hline
\end{tabular}


- Contenidos: Los contenidos son explicados a través de la lección magistral (86\%). La secuencia viene recogida en el libro de texto. Para el resto de docentes (14\%) los contenidos quedan recogidos en el currículo y se aplican a través de proyectos Por ejemplo; Los Miniuns. En concreto estos docentes son especialistas y no generalistas (profesor de Educación Física y Educación Musical). Todos los docentes tienen marcada su planificación en el aula a través de sus programaciones.

- Evaluación: La evaluación es compleja en esta etapa. Ésta depende del contexto y de los instrumentos utilizados por el docente. Por ello cuestiones como que se evalúa, con qué instrumentos, cuando se lleva a cabo son elementos relevantes para el docente. El $100 \%$ de los entrevistados utilizan listas de control, encuestas, trabajos individuales y trabajos en grupo como principal forma de evaluación al discente. De los 14 docentes, 12 utilizan examen como forma de evaluación. Es un dato relevante teniendo en cuenta que los dos docentes que no las utilizan son los profesores que trabajan por proyectos en el aula. En menor medida se realiza a través de la escala de estimación y del portafolio, 6 y 7 personas, respectivamente. La entrevista sólo es utilizada por 3 docentes al igual que los mapas conceptuales y resolución de casos concretos. Éstas últimas recogidas en la categoría de otros. Sólo un profesor utiliza el ejercicio práctico como forma de evaluación (figura 2).

Figura 2

Instrumentos de evaluación de la práctica docente

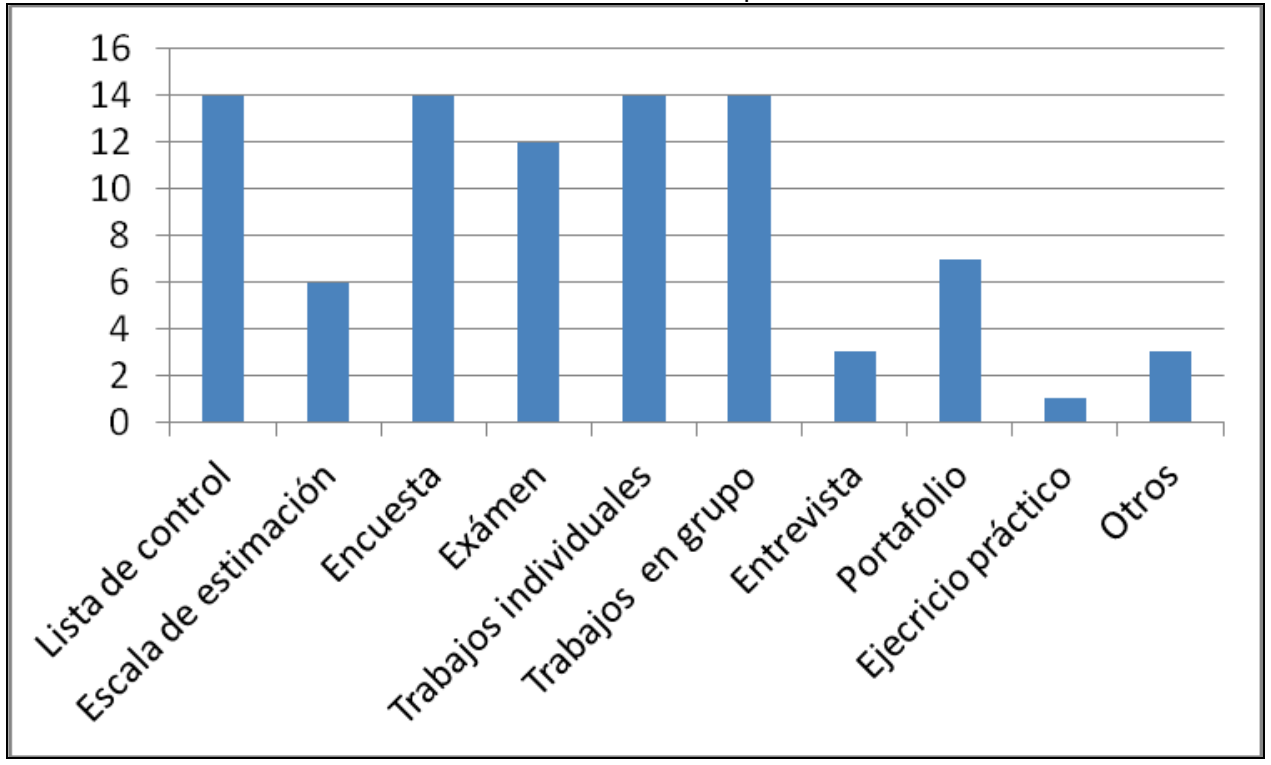

- Recursos: Dos de los catorce docentes no lleva el libro de texto como guía de su asignatura. Los materiales son realizados por ellos mismos. El resto utilizan libros de editoriales Santillana y Anaya. Todos los docentes afirman utilizar el aula Plumier priorizando la importancia de las tecnologías de la información.

\section{Discusión}

Con esta investigación se ha analizado el conocimiento y aplicación de las competencias y metodologías aplicadas en un centro de Primaria. Se ha comprobado que estamos en el camino de una renovación educativa. Ya hay docentes que trabajan por proyectos en donde las metodologías se basan esencialmente en que el alumno tenga un papel activo. En el siglo XXI se plantea como cambio esencial las renovaciones metodológicas en donde el docente pasa a ocupar un papel secundario y el alumno un papel esencial en la construcción de su propio conocimiento. El 
profesorado debe de ayudar al alumnado en su aprendizaje y enseñarle en base a sus particularidades.

El análisis de la realidad educativa es un aspecto esencial en el avance del sistema educativo. Es necesario pararse a pensar y determinar que hacemos y hacia dónde vamos ya que la mejora de la calidad educativa dependerá del nivel profesional de los docentes.

En el ámbito de la renovación educativa nos encontramos con la incorporación de las competencias. Casi la totalidad de docentes señalan que se está hablando del mismo perro aunque de distinto collar confundiéndose estás con los temas transversales, a pesar de ser docentes jóvenes. Estudios como los de Méndez, Giménez y Rio (2015) analizan y valoran la incorporación de las competencias básicas en la labor diaria de los docentes de Educación Primaria de la comunidad autónoma uniprovincial de España. El objetivo era conocer si los maestros incorporaban las competencias básicas a sus programaciones y cómo las llevaban a cabo o el estudio de Sarramona (2014) que analiza las competencias en función de su ámbito de aplicación. En concreto es una experiencia de integración de las competencias básicas en el currículum con la novedad de su clara formulación vinculadas a diferentes áreas 0 materias así como su gradación en diferentes niveles de consecución.

La gran mayoría de los docentes dicen que las competencias son conseguidas a través de los objetivos, pero no saben especificar o concretar una competencia concreta, lo confunden con temas transversales. Especifican que se habla del mismo concepto pero con distinto término, al igual que en el estudio de Ros ét al (2008), llegando a la conclusión de que a lo largo de su docencia siempre ha existido este tema y que lo único que hacen es cambiarle el nombre. Sólo dos de los docentes detallan las 8 competencias. Los datos recogidos aportan información relevante sobre el desarrollo integral del discente. En palabras de los docentes.

Este trabajo es una evidencia más de la heterogeneidad y complementariedad de las metodologías aplicadas en el aula. Sirva de ejemplo la catalogación de las metodologías (autónomas, grupales y expositivas) que realizan Brown y Atkins (en Fernández March, 2006), estableciendo una línea continua en donde en un extremo registran a las lecciones magistrales mientras que, en el lado opuesto, reflejan el trabajo autónomo que realiza el alumno, pasando por los trabajos en grupo. En la práctica existen diferentes métodos de enseñanza, aplicables en función de los objetivos que se persigan. En la actualidad, el docente debe de desarrollar estrategias de aprendizaje que promuevan la participación activa del alumno en el aula y como consecuencia el aprendizaje significativo (Faure, 1981; Collados, 2007)

La formación del profesorado es un desafío para las instituciones educativas. La necesidad de innovar, solucionar problemas y de adaptar al alumnado al contexto requiere de un gran avance para los planes educativos. Por tanto, no cabe duda de que la formación permanente del profesorado es el eje esencial por donde avanzar en el proceso educativo. Educar diferente requiere de una remodelación en la estructura organizativa de la escuela y en el modelo de educación. Esta necesita otro punto de partida, otros presupuestos antropológicos y éticos que hagan posible entender y hacer la educación de "otra manera" (Ortega \& Hernández 2008).

\section{Referencias}

Collados, EA. (2007). Creatividad y cooperación: un caso práctico de aprendizaje basado en problemas aplicado al diseño gráfico. Aula de innovación Educativa, 172, 40-4. 
Faure, G. (1981). Juego dramático en la escuela. Cuadernos de Pedagogía, 85, 25-28.

Fernández March, A. (2006). Metodologías activas para la formación de competencias. Valencia: ICE. Universidad Politécnica de Valencia

Herrán, A. (2008). Didáctica general. La práctica de la enseñanza en la Educación Infantil, Primaria y Secundaria. Madrid: Mc Graw-Hill.

Ley Orgánica 2/2006, de 3 de mayo, de Educación, modificada por la Ley Orgánica 8/2013, de 9 de diciembre, para la mejora de la calidad educativa.

Méndez, D., Gimeno, A. y Rio, FJ. (2015). Análisis y valoración del proceso de incorporación de las Competencias Básicas en Educación Primaria. Revista de Investigación Educativa, 33(1), 233-246.

Ortega, P. y Hernández Prados, M.A. (2008). Lectura, narración y experiencia en la educación en valores. Revista Iberoamericana de Educación, 45, 1-5.

Real Decreto 1513/2006 por el que se establecen las Enseñanzas Mínimas de Educación Primaria.

Ros, R., Alfageme, MB. y Vallejo, M. (2008). Enfoques de enseñanza en un centro de Primaria: cambio o continuidad. Servicio de publicaciones Universidad de Murcia. II Jornadas de los Máster en Investigación e Innovación en Educación Infantil y Educación Primaria.

Sarranova, J. (2014). Competencias básicas y curriculum. El caso de Cataluña. Ediciones Universidad de Salamanca, 3, 205-228.

Tobon, S. (2006). Aspectos básicos de la formación basada en competencias. Talca: Proyecto Mesesup.

\section{Anexo I}

\section{Protocolo de la entrevista}

Al inicio de la entrevista se informa al entrevistado sobre el objeto de la misma, dejando constancia del tratamiento globalizado de los datos y la privacidad de los mismos.

\section{MARQUE CON UNA (X) DONDE CORRESPONDA:}

SEXO:

$\begin{array}{ll}\text { a } & \text { Mujer } \\ \text { a } & \text { Hombre }\end{array}$

SITUACION LABORAL:

a Fijo.

a Temporal (Interinidad)
EDAD:

$\begin{array}{ll}\square & 20 \text { a } 30 \\ \square & 31 \text { a } 40 \\ \square & 41 \text { a } 50 \\ \square & 51 \text { a } 60 \\ \square & \text { Más de } 61\end{array}$

AÑOS DE DOCENCIA EJERCIDA EN EL CENTRO:

$\begin{array}{ll}\square & 1 \text { a } 5 \text { años. } \\ \square & 5 \text { a } 10 \text { años. } \\ \square & 10 \text { a } 15 \text { años. } \\ \square & 15 \text { a } 20 \text { años. } \\ \square & 20 \text { a } 25 \text { años. }\end{array}$

- Más de 25 años. 
NIVEL EDUCATIVO:

口 Diplomatura, Grado

- Licenciatura

- Máster

a Doctorado

\section{ESPECIDALIDAD QUE IMPARTE:}

\section{口 Primaria}

口 $\quad$ Educación Infantil

a Educación Física

a Educación Especial

a Educación Musical

a Lenguas Extranjeras

a Audición y Lenguaje.

a Otros
HORAS A LA SEMANA TRABAJADAS:

- De 1-20 Horas semanales.

a De 20-30 horas semanales.

a De 30-40 horas semanales.

CURSO AL QUE IMPARTE CLASE

$\begin{array}{ll}\square & 1^{\circ} \\ \square & 2^{\circ} \\ \square & 3^{\circ} \\ \square & 4^{\circ} \\ \square & 5^{\circ} \\ \square & 6^{\circ}\end{array}$

\section{PROTOCOLO}

\section{Competencias}

$>$ ¿Qué piensas sobre el tema de las competencias que se están empezando a introducir en el sistema educativo por medio del Espacio Europeo de Educación? (¿Da un nuevo sentido a la enseñanza? ¿Y al aprendizaje?)

$>$ ¿Conoces las competencias?

\section{Metodologías}

$>$ ¿Cómo trabajas con tus alumnos / as? ¿Qué metodologías o técnicas aplicas en el aula? Especifica cuál.

\begin{tabular}{ll} 
& \multicolumn{1}{c}{ GRUPAL } \\
$\square$ & Caso dramatizado \\
$\square$ & Debate \\
$\square$ & Aprendizaje basado en problemas \\
$\square$ & Trabajo dirigido \\
$\square$ & Juegos educativos \\
$\square$ & Dinámica de grupo \\
$\square$ & Juego de roles \\
$\square$ & Resolución de ejercicios \\
$\square$ & Otros
\end{tabular}

\begin{tabular}{ll} 
& \multicolumn{1}{c}{ INDIVIDUAL } \\
$\square$ & Enseñanza a distancia \\
$\square$ & Contrato de aprendizaje \\
$\square$ & Enseñanza personalizada \\
$\square$ & Aprendizaje orientado a proyectos \\
$\square$ & Programa de lecturas \\
$\square$ & Enseñanza modular \\
$\square$ & Enseñanza a distancia \\
$\square$ & Enseñanza programada \\
$\square$ & Otros
\end{tabular}

$>$ ¿De qué depende la utilización de unas u otras?

$>$ ¿Cómo organizas el tiempo y el espacio?

$>$ ¿Cómo promueves la participación entre tus alumnos? ¿Y la interacción contigo?

\section{Contenidos}

$>$ ¿Estableces una secuenciación determinada?

$>$ ¿Cuál sería? ¿Qué criterios tienes en cuenta?

$>$ Sigues habitualmente algún libro de texto? ¿Cuál?

\section{Evaluación}

$>$ ¿Cuándo evalúas y cómo lo realizas?

$>$ ¿Cuándo? ¿Con qué instrumentos?

$>$ Para ti, ¿Cuál es la importancia de este proceso? 